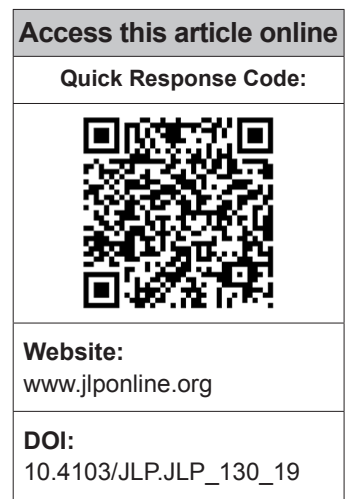

Department of Pathology,

Bankura Sammilani Medical College,

${ }^{1}$ Department of Pathology, Malda Medical College, Kolkata, West Bengal, India

Address for correspondence:

Dr. Saikat Mandal, Department of Pathology, Malda Medical College, Malda, West Bengal, India. E-mail: saikat1607@ gmail.com

Submission: 14-08-2019 Accepted: 21-08-2019

\title{
Expression of p57 immunomarker in the classification and differential diagnosis of partial and complete hydatidiform moles
}

\author{
Santosh Kumar Mondal, Saikat Mandal ${ }^{1}$, Saptarshi Bhattacharya, \\ Utpal Kumar Panda, Arpan Ray, Sk. Moyaraf Ali
}

\section{Abstract:}

BACKGROUND: Hydatidiform mole (HM) is characterized histologically by cystic swelling of the chorionic villi, accompanied by variable trophoblastic proliferation. The most important reason for the correct recognition of moles is that they are associated with an increased risk of persistent trophoblastic disease (invasive mole) or choriocarcinoma.

AIMS AND OBJECTIVES: The aim of the study was to determine whether there is any role of p57 in differentiating partial and complete moles by immunohistochemical staining.

MATERIALS AND METHODS: A prospective observational study was undertaken in which 40 cases of molar pregnancy included over a period of 2 years. Detailed clinical and family histories were obtained from each patient. Histopathological examination followed by immunohistochemical study with p57 done in each case. Ultrasonography findings and serial titers of serum beta-human chorionic gonadotropin were noted whenever necessary.

RESULTS: Among the forty cases included, 25 (62\%) had complete molar (CM) pregnancy, whereas the rest $15(38 \%)$ had partial mole (PM). Both $\mathrm{CM}$ and PM were more pronounced in the age group of $20-25$ years ( $44 \%$ and $60 \%$, respectively), and among nulliparous women (68\% and $70 \%$ respectively), 17 (42.5\%) mothers had a prior history of abortion. In the histologically unequivocal cases of complete mole, $96 \%$ (24 of 25) did not express p57 and a single case was focal positive. In contrast, it was strongly and continuously expressed in both villous cytotrophoblast and stromal cells in all cases of PM (15 of 15).

CONCLUSION: p57 immunomarker is very helpful to diagnose and differentiate complete and partial HM.

Key words:

Hydatidiform mole, immunohistochemistry, P57

\section{Introduction}

Gis estational trophoblastic disease (GTD) $\checkmark$ is a group of diseases related to normal or abnormal gestation that has as a common denominator the proliferation of trophoblast. The individual disorders differ remarkably in appearance and clinical significance.

This is an open access journal, and articles are distributed under the terms of the Creative Commons Attribution-NonCommercial-ShareAlike 4.0 License, which allows others to remix, tweak, and build upon the work non-commercially, as long as appropriate credit is given and the new creations are licensed under the identical terms.

For reprints contact: reprints@medknow.com
The major diseases are hydatidiform mole (HM) (complete, partial, or invasive), placental site trophoblastic tumor, and choriocarcinoma. The pathogenesis of these remains poorly understood, but genomic imprinting is believed to play a key role in the formation of HMs. ${ }^{[1,2]}$

There is a striking geographic variation in the frequency of complete mole. The incidence of 1 in 2000 deliveries reported in

How to cite this article: Mondal SK, Mandal S,
Bhattacharya S, Panda UK, Ray A, Ali SM. Expression
of p57 immunomarker in the classification and
differential diagnosis of partial and complete
hydatidiform moles. J Lab Physicians 2019;11:270-4. 
the classic studies by Hertig represents an average for young healthy women in the United States. In Southeast Asia, the reported incidence is at least four to five times greater. Yet, higher incidences have been reported from Mexico (1:200), the Philippines (1:173), India (1:160), Taiwan (1:125), and Indonesia (1:82). Approximately $0.2 \%$ to $5 \%$ of patients with a partial mole (PM) and $15 \%$ to $25 \%$ of patients with a complete molar (CM) will develop persistent GTD. Malignant transformation from persistent GTD to choriocarcinoma has been observed in $3 \%$ to $5 \%$ of patients with a CM. Choriocarcinoma has also been observed in patients following a PM, but this is extremely rare.

The gene p57kip2 (p57) is the protein product of the paternally imprinted but maternally expressed gene. CDKN1C is located on chromosome 11p15.5. ${ }^{[3]}$ As CMs lack a maternal genomic component, they are not expected to express imprinted genes that are normally expressed by the maternal allele, and immunohistochemical analysis for p57 has been shown to be a valuable tool in the diagnosis of a CM. ${ }^{[2]}$ However, immunohistochemical analysis for p57 cannot distinguish a PM from an HA. ${ }^{[3]}$

The present study attempted to find whether there is any role of p57 in differentiating PM and complete mole from the normal villous structure by immunohistochemical staining. If it is found that p57 immunomarker has a role, then we will try to find out whether this p57 immunomarker can be used as a diagnostic tool especially in case of any diagnostic dilemma during histopathological diagnoses of these entities.

\section{Materials and Methods}

This study was conducted in the department of pathology in collaboration of the department of gynecology and obstetrics in our tertiary care teaching medical institution over a period of 2 years from March 2017 to February 2019. Detailed history regarding the maternal age, prior history of any molar pregnancy, and gestational age at the time of evacuation were taken. The presenting complaints (bleeding per vagina, pain abdomen, something coming out per vagina etc.) were noted. Any previous history of infertility and history of oral contraceptive pill (OCP) were also taken. Ultrasonography and serum beta-human chorionic gonadotropin reports were reviewed when available.

After the expulsion of product of conception, it was kept in $10 \%$ formal saline. The specimen was grossed after allowing enough time for it to be fixed [Figure 1]. Sections were taken from appropriate areas, and they will be processed and stained with hematoxylin and eosin ( $\mathrm{H}$ and $\mathrm{E})$. The immunomarker used in our study was p57. The entire primary antibodies were mouse monoclonal antibodies (Leica). They were supplied prediluted and ready to use. The secondary antibody kits were supplied by NOVOCASTRA and were ready to use. Appropriate positive and negative control experiments were run with each case. P57 staining was considered negative when cytotrophoblasts and villous stromal cells showed $<10 \%$ nuclear immunoreactivity. Maternal decidua and proliferating trophoblasts served as internal control samples, and staining was necessary for the results to be considered valid.

Forty cases of HM were studied in our institution for a period of 1 year and 7 months from March 2017 to February 2019. Specimens received in our pathology department were subjected to histopathology examination to determine the histological type HM followed by immunohistochemical study to determine p57 expression. The collected data were tabulated and analyzed. On routine histopathological examination, among the total of 40 cases, 25 cases were diagnosed as complete mole $(62 \%)$ and the rest $15(38 \%)$ cases were PM [Figure 2].

Seventy-five percent (30) of the patients were in low socioeconomic group and $20 \%$ (8) from middle and only $5 \%$ from the upper class. The mean age of the patients was $23.47 \pm 4.7$ years (range: $17-36$ years), and the mean age of diagnosis was $13.12 \pm 4.61$ weeks (range: $6-23$ weeks).

Forty percent (16) of the patient's blood group were $\mathrm{B}+\mathrm{ve}, 30 \%$ of the patients were $\mathrm{O}+\mathrm{ve}$ (12 cases), $25 \%$ were $A+v e$ (ten), and $5 \%$ were $A B+v e$. None of them were negative blood group. $\mathrm{B}+$ ve blood group was most common among both the groups (nine patients in each group). $\mathrm{AB}+\mathrm{ve}$ group was least common in all the groups (single patient in each group). Six patients having $\mathrm{CM}$ had A+ve blood group and nine had $\mathrm{O}+$ ve blood group. Four and three patients with PM had A+ve and $\mathrm{O}+$ ve blood groups, respectively.

In the study population, $67.5 \%$ of the patients presented with amenorrhea and per vaginal bleeding. Seven patients $(17.5 \%)$ had symptoms of pain abdomen with certain period of amenorrhea. Ten percent of the patients had symptoms of both pain abdomen and per vaginal bleeding, whereas only two patients $(5 \%)$ perceived that something grape-like vesicles come out per vagina (both of them had CM). Sudden onset of pain in the abdomen following a period of amenorrhea was the most common presenting feature. Because as many as 17 patients with $\mathrm{CM}$ and ten patients with PM had this presenting feature.

\section{Results}

In our study, it was found that the most common age group which had HM is between 20 and 25 years (44\% of the 


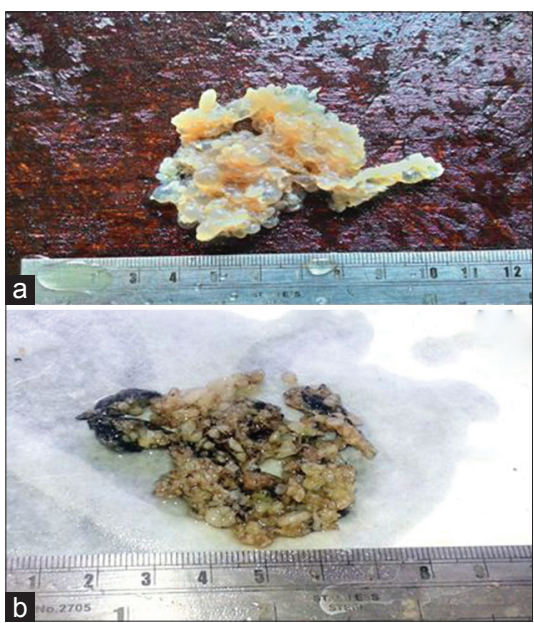

Figure 1: (a) Gross picture of complete mole, (b) Gross picture of partial mole

patients with $\mathrm{CM}$ and $60 \%$ of the patients having PM fell in this age group). Both PM and CM found to be declining as maternal age progresses; however, $\mathrm{CM}$ is more common than PM after $30 \mathrm{~s}$ as seen during the study (16\% in contrast to $13.3 \%$ in CM and PM, respectively). Chi-square test was done and $P=0.69$.

Hence, it is evident that most of the complete moles (52\%) and PMs (53.4\%) were evacuated in the late first trimester or early second trimester (10-15 weeks). Twenty percent of $\mathrm{PM}$ and $16 \%$ of $\mathrm{CM}$ were terminated in gestational age $<10$ weeks. Gestational age at the time of evacuation was between 16 and 20 weeks in seven of CMs and three of PMs. Only single case crosses gestational age of $>20$ weeks in both the groups. Chi-square test was done and $P=0.92$.

Hence, in this study, it was found that both complete mole (68\%) and PM (70\%) are more common among the nulliparous women and parous women more commonly present with complete mole (32\%) relative to PM (30\%). Chi-square test was performed and $P$ value calculated was 0.93 .

Hence, it is seen that most of our cases did not have any previous history of any previous abortion (56\% of complete moles and $60 \%$ of PMs); however, when there is a history of miscarriage previously, the incidence of complete mole is higher than PM ( $44 \%$ in contrast to $40 \%)$. Chi-square test was done and $P=0.80$.

It is found that total $12(30 \%)$ cases used OCP previously; among them, seven had complete mole and five PMs. It is also seen that PM is more related to OCP usage than complete mole $(33.33 \%$ in contrast to $28 \%)$. It is also found that complete mole is common among the patients who previously used as well as who did not use OCPs. Chi-square test was done and $P$ value calculated was 0.72 .

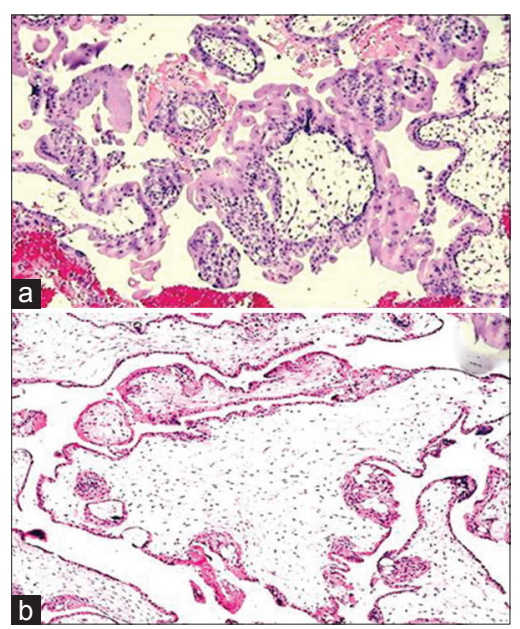

Figure 2: (a) Photomicrograph of complete mole showing circumferential proliferation of villous trophoblastic cells ( $\mathrm{H}$ and E, $\times 100$ ), (b) Photomicrograph of partial mole showing mixture of small villi and large hydropic villi with an irregular, scalloped outline ( $\mathrm{H}$ and $\mathrm{E}, \times 100)$

Our study includes 33 (82.5\%) cases that did not have any previous history of infertility. The occurrence of complete mole is more related to $\mathrm{PM}(20 \%$ and $13.5 \%$, respectively). Chi-square test was done, $P$ value revealed to be 0.59 .

After taking all relevant clinical data and reviewing the hematoxylin and eosin ( $\mathrm{H}$ and $\mathrm{E})$-stained sections, immunohistochemistry with p57 monoclonal primary antibody done using the standard immunoperoxidase method revealed that all 15 cases of PM show strong positivity in villous cytotrophoblast as well as in the stromal cells (15 of 15,100\%) [Figure 3].But 24 cases of 25 cases of histologically diagnosed complete mole showed negative immune stain. Single case of complete mole showed focal positivity with p57 immunohistochemistry. Single case of complete mole showed focal positivity with p57 immunohistochemistry [Figure 3].

Those cases which are strongly positive for p57 and are a case of PM are considered as true positive (TP), and true negative (TN) are those cases which are negative for $\mathrm{p} 57$ immunostain (i.e., complete mole). False positive (FP) cases were those $\mathrm{CMs}$ which were positive for $\mathrm{p} 57$. On the other hand, PMs which did not show p57 positivity (or negative) were considered as false negative (FN).The specificity of p57 immunomarker in properly diagnosing $\mathrm{HM}$ is $96 \%$, whereas the sensitivity calculated is $100 \%$ (as sensitivity $=\mathrm{TP} / \mathrm{TP}+\mathrm{FN}$ and specificity $=\mathrm{TN} / \mathrm{TN}+\mathrm{FP})$.

The specificity of p57 immunomarker in properly diagnosing $\mathrm{HM}$ is $96 \%$, whereas the sensitivity calculated is $100 \%$ (as sensitivity $=\mathrm{TP} / \mathrm{TP}+\mathrm{FN}$ and specificity $=\mathrm{TN} /$ $\mathrm{TN}+\mathrm{FP})$.

\section{Discussion}

$\mathrm{HM}$ is an abnormal placentation with hydropic swelling 


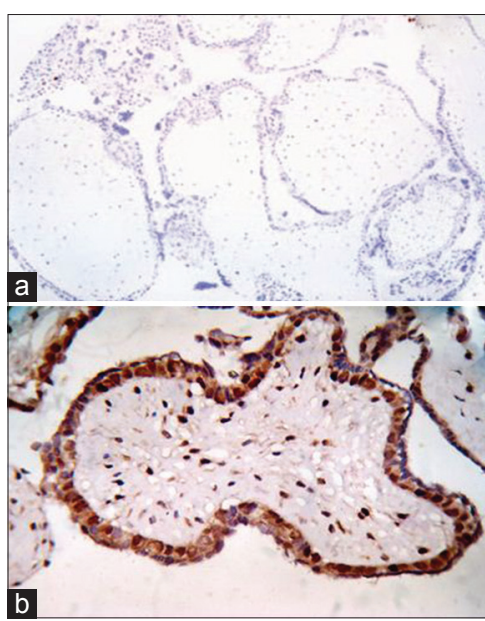

Figure 3: (a) Photomicrograph of complete mole showing complete absence of p57 immunostain in complete mole ( $\mathrm{H}$ and $\mathrm{E}, \times 100)$, (b) Photomicrograph of partial mole showing strong p57 positivity in villous cytotrophoblast as well as in the stromal cells $(p 57, \times 400)$

of chorionic villi and trophoblastic proliferation. All molar pregnancies result from abnormal fertilization. The pathogenesis of these remains poorly understood, but genomic imprinting is believed to play a key role in the formation of HMs. ${ }^{[1,2]}$ In our study of the 40 cases diagnosed as molar pregnancies by hematoxylin and eosin, the actual age range was from 17 to 36 years; the age distributions were mean: 23 , median: 22 , and mode: 22 years; the majority of the cases $(50 \%)$ were between the age of 20 and 25 years ; and the correlation between the age of the patients and the type of the HM was not significant.

In a similar study, Shakil H. Merchant et al., in the USA, found that patients' age ranged from 14 to 41 years; the mean age was 24.3 years. Dr. Rehab K. Abbas. and Dr. Khitam R. Al-Khafaji in their study in Baghdad university found that the age distributions were mean: 29 , median: 27 , mode: 20 years, and the majority of the cases (50\%) were between the age of 20 and 29 years. In both the studies, there was no correlation between age of the patients and the disease. The incidence of CM and PM in our study was $62 \%$ and $38 \%$, respectively. A 5-year study done by AA Mayun found such incidence of CM and PM (52.9\% and $47.15 \%$, respectively). In a study between January 2011 and December 2015 byLelic $\mathrm{M}$ et al., there were 198 cases of histologically confirmed HM, 185 PM, $12 \mathrm{CM}$, and one case of undefined HM. They found mean maternal age in the CM group was 24.7 years and in the PM group was 26.9 years, with no significant differences among these two groups $(P=0.27) \cdot{ }^{[4]}$

Madi JM et al. undertook a systemic review and meta-analysis to see the accuracy of p57kip2 compared with genotyping to diagnose complete HM. Their bivariate meta-analysis produced a summary sensitivity of 0.984 (95\% confidence interval [CI]: 0.916-1.000) and specificity of 0.625 (95\% CI: $0.503-0.736)$ with significant heterogeneity for specificity $\left(I^{2}=71.8\right.$, Chi-square $P=0.029$ ). The pooled summary diagnostic odds ratio was 56.54 (95\% CI: 11.03-289.74) with no heterogeneity $\left(I^{2}=0.00 \%\right.$, Chi-square $\left.P=0.67\right)$. The diagnostic performance of the test was high with an area under the curve of $0.980 .{ }^{[5]}$

Erol O et al. compared p57, c-erbB-2, CD117, and Bcl-2 immunomarker expression in the differential diagnosis of HM and hydropic abortion. They found immunohistochemical examination of p57, c-erbB-2, CD 117, and Bcl-2 expression represented a relatively simple, reliable, and cost-efficient procedure to definitively distinguish among CM, PM, and hydropic abortus (HA). ${ }^{[6]}$ In a study, Triratanachat $\mathrm{S}$ et al. found that the sensitivity of final $\mathrm{H}$ and $\mathrm{E}$ diagnosis for $\mathrm{CM}$ was $89.7 \%$ and the specificity was $95.0 \%$. PM sensitivity and specificity of final $\mathrm{H}$ and $\mathrm{E}$ diagnosis was $95.0 \%$ and $89.7 \%$, respectively. They opined that histopathological diagnosis alone has certain limitations in accurately defining types of HM; P57KIP2 immunohistochemistry is practical and can be a useful adjunct to histopathology to distinguish $\mathrm{CM}$ from non-CM. ${ }^{[7]}$

In our current study, we found that $100 \%$ (15 of 15) of the PMs were strong positive for p57 immunostain. However, $96 \%$ (24 of 25) cases of complete mole did not take p57 staining and $4 \%$ (one case) stained with focal positivity. Shigeru Sasaki et al. studied 14 cases of products of conception, among which four did not take any p57 stain and were diagnosed as CM. Rest of the cases stained diffusely although they cannot differentiate between PM and HA. Shakil H. Merchant et al. compared the use of p57KIP2 staining in the differential diagnosis of 68 morphologically challenging cases of early first trimester hydropic placentas. Concordant results were obtained in 65 of 68 cases studied. In 2 of 3 cases with a discordant diagnosis, microsatellite DNA genotyping analysis agreed with the results of p57KIP2 staining, confirming that positive p57KIP2 staining is a highly sensitive and specific marker for excluding CM in their study. LeGallo et al. found that a combination of immunohistochemical analysis for p57 and HER2 FISH (or potentially other probes) will aid in the accuracy in identifying molar pregnancies. The absence of p57 staining is specific for CM. Landolsi et al. evaluated the results of routine histopathological examination and p57KIP2 immunoreactivity in a large series of $132 \mathrm{CM}$, $49 \mathrm{PM}$, and $39 \mathrm{HA}$ and obtained concordant results in $210(95.45 \%)$ cases which is very close to our findings.

Distinction of HMs from nonmolar specimens and subclassification of HMs as complete HM versus partial HM are important for clinical practice and 
investigational studies. Ronnett and Colgan et al. explained how ancillary techniques target the unique genetic features of HMs to establish diagnostic truth and highlighted the issue of diagnostic reproducibility. ${ }^{[8,9]}$ They explained the importance of diagnostic accuracy and illustrated the use of p57 immunohistochemistry and polymerase chain reaction-based DNA genotyping for diagnosis. Genotyping, which compares villous and decidual DNA patterns to determine the parental source and ratios of polymorphic alleles, distinguishes purely androgenetic complete HMs from diandric triploid partial HMs, and both of these from biparental nonmolar specimens. They opined that an algorithmic approach using these techniques should be advocated for diagnosis of these cases. ${ }^{[8,9]}$ In a recent study, differential expression of Twist1, Ki-67, and E-cadherin was analyzed by Moussa et al., in gestational products from 55 cases of CM, PM, and HA using immunohistochemistry ${ }^{[10]}$ In their study, they found Twist1 expression was a highly reliable marker for the diagnosis of CM, whereas combined Ki-67 and E-cadherin immunoreactivity could distinguish PHM from nonmolar pregnancies. ${ }^{[10]}$

Diagnosis and classification of molar gestations into complete mole and PM and their differentiation from nonmolar hydropic abortions are traditionally accomplished by morphology alone. The severity of the changes varies considerably from case to case and from villous to villous and may be difficult to appreciate in very early cases, as evidenced by the high degree of inter- and intraobserver variability. In such challenging cases, p57 immunohistochemistry can play a pivotal role which may be more strengthened with ploidy analysis.

\section{Financial support and sponsorship} Nil.

\section{Conflicts of interest}

There are no conflicts of interest.

\section{References}

1. Hui P, Buza N, Murphy KM, Ronnett BM. Hydatidiform moles: Genetic basis and precision diagnosis. Annu Rev Pathol 2017;12:449-85.

2. Samadder A, Kar R. Utility of p57 immunohistochemistry in differentiating between complete mole, partial mole and non-molar or hydropic abortus. Indian J Med Res 2017;145:133-7.

3. Lelic M, Fatusic Z, Iljazovic E, RamicS, Markovic S, Alicelebic S, et al. Challenges in the routine praxis diagnosis of hydatidiform mole: A Tertiary health center experience. Med Arch 2017;71:256-60.

4. Madi JM, Braga A, Paganella MP, Litvin IE, Wendland EM. Accuracy of p57KIP2 compared with genotyping to diagnose complete hydatidiform mole: A systematic review and meta-analysis. BJOG 2018;125:1226-33.

5. Erol O, Suren D, Tutus B, Yararbas K, Sayiner A, Ozel MK, et al. Comparison of p57, c-erbB-2, CD117, and bcl-2 expression in the differential diagnosis of hydatidiform mole and hydropic abortion. Eur J Gynaecol Oncol 2016;37:522-9.

6. Triratanachat $S$, Nakaporntham $P$, Tantbirojn $P$, Shuangshoti $S$, Lertkhachonsuk R. Role of P57KIP2 immunohistochemical expression in histological diagnosis of hydatidiform moles. Asian Pac J Cancer Prev 2016;17:2061-6.

7. Ronnett BM. Hydatidiform moles: Ancillary techniques to refine diagnosis. Arch Pathol Lab Med 2018;142:1485-502.

8. Luchini C, Chilosi M, Manfrin E. The importance of immunohistochemistry in the differential diagnosis of molar disease. Pathologica 2016;108:151-3.

9. Colgan TJ, Noor A, Nanji S, Chang MC, Kolomietz E. Molecular diagnosis of placental hydatidiform mole: Innovation and outcomes. J Obstet Gynaecol Can 2017;39:1049-52.

10. Moussa RA, Eesa AN, Abdallah ZF, Abdelmeged A, Mahran A, Bahaa $\mathrm{H}$, et al. Diagnostic utility of twist1, ki-67, and E-cadherin in diagnosing molar gestations and hydropic abortions. Am J Clin Pathol 2018;149:442-55. 